\title{
Editorial
}

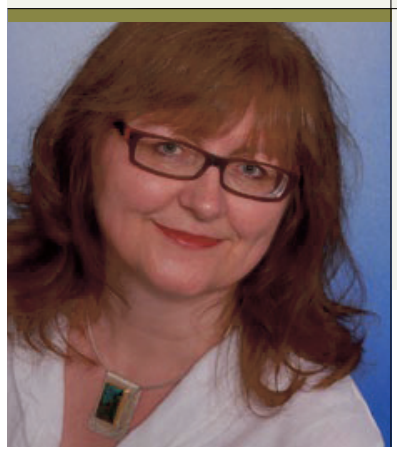

"Schmerztherapie und Palliativmedizin sind eine

interdisziplinäre Aufgabe."

Doris Berger,

Redaktion Angewandte Schmerztherapie und Palliativmedizin

\section{Quervernetzt fortbilden}

,D aher ist jeder Arzt verpflichtet, sich auf diesem Gebiet, das in der universitären Ausbildung bisher keinen Platz gefunden hat, fortzubilden und sich über moderne Verfahren der Schmerzbehandlung umfassend zu unterrichten. Er muss sie entweder selbst beherrschen oder so viel von ihnen verstehen, dass er den Schmerzkranken an einen geeigneten Spezialisten oder eine fachkundige Einrichtung überweisen kann", zitiert Michael Zenz in seinem Editorial der aktuellen Ausgabe der Zeitschrift „Der Schmerz" den Juristen Klaus Kutzer. Kutzer, der von 1982 bis 2001 Richter am Bundesgerichtshof war, schrieb diese Worte bereits 1991 [Schmerz 1991; 5(2):53-5]. Und sein Anliegen ist aktuell wie vor 20 Jahren. Noch immer steht dem Schmerzkranken keine "lautstarke Lobby" zur Seite, noch immer ist die Schmerztherapie kein integrierter Bestandteil der universitären Ausbildung, auch wenn es zahlreiche Fort-und Weiterbildungsangebote gibt.

Dennoch - eine adäquate Schmerzbehandlung, insbesondere von chronischen Schmerzen und von Tumorschmerzen, ist ebenso wie die Palliativmedizin Anliegen vieler: der Allgemeinmediziner, der Anästhesisten, der Orthopäden, der Onkologen, der Palliativmediziner und vieler anderer mehr. Dies spiegelt die gesamte Breite der Interdisziplinarität der Schmerztherapie und Palliativmedizin wider. Die Interdisziplinarität bezieht sich aber nicht nur auf die ärztliche Versorgung. Gerade im Bereich Schmerztherapie und Palliativmedizin handelt es sich um eine gesamtgesellschaftliche Aufgabe, die es zu bewältigen gilt.
Als Fachverlag unterstützt SpringerMedizin die Ärzteschaft mit einer Vielzahl an Publikationen zum Thema Schmerzbehandlung und Palliativmedizin. Seit vier Jahren auch mit der Zeitschrift „Angewandte Schmerztherapie und Palliativmedizin“. Seit Mitte vergangenen Jahres zudem durch das Internet-Portal www.SpringerMedizin.de. Nach der kostenlosen Registrierung stehen dem Nutzer Nachrichten und Hintergrundbeiträge aus allen Fachgebieten, aus Gesundheitspolitik und -ökonomie zur Verfügung. Gerade die quervernetzte Informationsvermittlung ist ein Pluspunkt des Portals.

Anfang des Jahres wurden die Zeitschriften-Homepages, auch die von „Angewandte Schmerztherapie und Palliativmedizin", in das Internet-Portal integriert. Damit steht dem Abonnenten der Zeitschrift auch der Mehrwert von SpringerMedizin.de zur Verfügung. Neben dem Vollzugriff auf die Inhalte von „Angewandte Schmerztherapie und Palliativmedizin" finden sich hier viele weitere Informationen.

\section{Ihre}

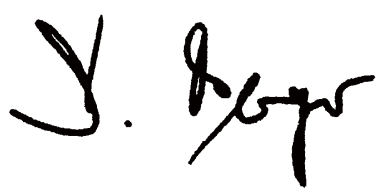

